\section{The role of rectus muscle myectomy in the management of large-angle strabismus for Graves' ophthalmopathy}

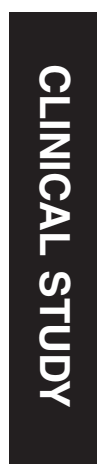

\author{
Abstract \\ Purpose Retrospective noncomparative case \\ series to investigate the role of rectus muscle \\ myectomy for the treatment of large-angle \\ strabismus in patients with Graves' \\ ophthalmopathy. \\ Patients and methods Data from 47 \\ consecutive patients with Graves' \\ ophthalmopathy who underwent complete \\ myectomy for large-angle strabismus \\ (strabismus greater than 25 prism diopters \\ (PDs)) were collected retrospectively. Pre- and \\ postoperative deviations in primary and \\ reading position were measured in PDs. \\ Postoperative deviation of $<5$ diopters in \\ primary gaze and functional binocular vision \\ in central $30^{\circ}$ field were considered as \\ successful surgical outcomes. \\ Results Patients undergoing complete \\ myectomy of the restricted muscles in large- \\ angle strabismus achieved a $78.7 \%$ success \\ rate after the first surgery. Reoperation \\ performed on seven patients resulted in \\ $85.7 \%$ success rate in reoperation group. The \\ overall success rate was $\mathbf{9 1 . 5 \%}$. The mean \\ efficacy of the isolated rectus muscle \\ myectomy was $34.3 \pm 7.7$ PDs. \\ Conclusions The complete rectus muscle \\ myectomy technique is effective and \\ predictable in the treatment of large-angle \\ strabismus in patients with Graves' \\ ophthalmopathy. \\ Eye (2017) 31, 1027-1033; doi:10.1038/eye.2017.28; \\ published online 3 March 2017
}

\section{Introduction}

The manifestations of Graves' ophthalmopathy include lid retraction, proptosis, soft tissue swelling, restrictive myopathy, and compressive optic neuropathy. ${ }^{1}$ Infiltration of lymphocytes

SL Liao ${ }^{1,2}$, Y-H Wei ${ }^{1}$ and AY-C Chuang ${ }^{3}$ and fibroblasts within the extraocular muscles and subsequent fibrosis result in restrictive strabismus and diplopia. ${ }^{2}$ Usually, most commonly involved extraocular muscles are the inferior and medial recti causing hypotropia and esotropia, respectively. ${ }^{3}$ In general, $~ 4-29 \%$ of patients with Graves' ophthalmopathy may require surgical correction. ${ }^{4-6}$

It is well accepted that the surgical management for large-angle deviation is much more challenging and is associated with higher failures rate. ${ }^{3-5,7,8}$ Extensively fibrotic and severely restricted extraocular muscles limit the postoperative predictability. For large-angle strabismus, especially with single-rectus muscle restriction without much of the contralateral antagonist muscle involvement, surgical approaches have mainly been multiple-muscle recession with adjustable sutures. ${ }^{7,9-13}$ The results of surgery are highly variable, with a reported success rate ranging from 47 to $81 \%$. $^{3,4,14,15}$ Aside from adjustable sutures, various techniques including tenon recession, ${ }^{16}$ relaxed muscle positioning technique, ${ }^{17}$ and correction of ductions ${ }^{6}$ have been advocated aiming to improve surgical outcomes. Despite the use of adjustable sutures, high reoperation rates ensue, ranging from 17 to $45 \%$ of cases. ${ }^{4,6,7,9-12,16,17}$

We herein present a novel approach to largeangle Graves'-related strabismus utilizing complete myectomy of the involved rectus muscles. The concept behind this approach was based on personal experience of SLL. In advanced Graves' orbitopathy, limited orbital space is often encountered, and the enlarged muscles were tightly pushed against the orbital wall in that adjustable sutures may not provide enough release. Even though the adjustable sutures for the correction of small-angle Graves'related strabismus in our experience have mostly
${ }^{1}$ Department of Ophthalmology, National Taiwan University Hospital, Taipei, Taiwan

${ }^{2}$ National Taiwan University, College of Medicine, Taipei, Taiwan

${ }^{3}$ Department of Ophthalmology, Mackay Memorial Hospital, Taipei, Taiwan

Correspondence: AY-C Chuang, Department of Ophthalmology, Mackay Memorial Hospital, 92, Chung-Shan N. Rd., Taipei 10449, Taiwan

Tel: +8862 25433535 ext 3179;

Fax: +8862 28342257.

E-mail: eyeann@gmail.com

Received: 17 November 2016

Accepted in revised form: 17 January 2017 Published online: 3 March 2017 
been successful, the results in large-angle strabismus were less satisfactory. Postoperative adjustments in these cases often involve total release of the sutures in order to achieve an end stage of no diplopia. At times, even such extreme maneuver was still inadequate. Our approach with myectomy performed at the bulk of the muscle belly was aimed to provide a quick and direct release of the poorly elastic muscle. We believe that the poorly elastic muscles will rest at its most relaxed position after the detachment and self-reattachment should provide sufficient force to maintain the muscle at the new position.

\section{Patients and methods}

Forty-seven consecutive patients with large-angle strabismus underwent rectus muscle myectomy by one surgeon (SLL) between 2002 and 2013 in National Taiwan University Hospital. Large-angle strabismus was defined as strabismus $>25$ prism diopters (PD). There were 17 males $(36 \%)$ and 30 females $(64 \%)$ with the median age of 56.7 years, ranging from 31 to 78 years. None of the patients received prior orbital decompression and the patients' strabismus conditions were considered stable for at least 3 months before the operation. All patients had normal thyroid function at the time of surgery. The mean time period between the initial clinic visit and the first surgery was $6.69 \pm 3.74$ months. The patients were followed for a mean period of $25.73 \pm 20.51$ months (ranging from 8.4 to 79.7 months). Institutional review board approval from National Taiwan University Hospital was obtained for the study. The study followed the recommendations of the Declaration of Helsinki and written informed consent was obtained from each patient.

All patients underwent computed tomographic (CT) imaging study before the muscle surgery. Extraocular muscle movements and forced duction test were evaluated for all patients. Severely restricted muscles were defined as positive forced duction test (with severely restricted movement toward the contralateral side) and marked extraocular muscle enlargement on CT image. Complete rectus muscle myectomy was reserved for severely restricted muscles regardless of the actual deviation. However, only those patients with strabismus $>25$ PD who underwent rectus muscle myectomy were included in the study. Patients with $<25-\mathrm{PD}$ strabismus were excluded despite bilateral severe upgaze limitation caused by asymmetric inferior rectus involvement (Table 1).

The calculation on the effect of single-muscle myectomy only included those successful cases undergoing one muscle surgery. In addition, Hertel exophthalmometry was also documented before muscle surgeries and 6 months after muscle surgeries.

\section{Surgical technique}

The surgeries were performed under general anesthesia. After the muscle was isolated and secured with a muscle hook, the inner blade of a Westcott Scissors was inserted under the muscle tendon to a depth of at least $10 \mathrm{~mm}$ from the muscle insertion. Care was taken not to compress or damage the underlying sclera since the muscle is extremely tight. After enough muscle was collected between the blades of the Westcott Scissors, one snip procedure was done to detach the entire muscle at the muscle belly (Figure 1). The entire muscle stump was retrieved and removed from the insertion (Figure 1). The conjunctiva was secured to the globe near the muscle insertion. Simultaneous detachment of the capsulopalpebral head was performed with inferior rectus muscle myectomy in order to minimize lower lid retraction. ${ }^{18}$ For patients who underwent re-detachment of the same muscles, we can usually find the muscle insertion located $\sim 8-10 \mathrm{~mm}$ below the original insertion. After the muscle was isolated and secured with a muscle hook, the inner blade of a Westcott Scissors was inserted under the muscle to a depth as far as possible. The entire muscle stump was detached and removed from the insertion.

Surgical success was defined as less than 5-PD deviation in primary and reading position and no diplopia within central $30^{\circ}$ field. Patients with persistent diplopia were considered as surgical failure. The patients who underwent second operations were further grouped into under-correction and overcorrection groups.

Reoperation for under-correction included re-detachment of the same muscle and/or additional recession/ myectomy of the antagonist of the yoke muscle. Reoperation for overcorrection involved recession/ myectomy of the antagonist in the same eye.

Preoperatively and postoperatively exophthalmometry was documented in 35 patients. The data were analyzed with paired $t$-test.

\section{Results}

Forty-seven patients underwent rectus muscle myectomy for the correction of large-angle strabismus related to Graves' disease. A summary of the types of surgical procedures is listed in Table 1 . The majority $(n=42$, $89.4 \%$ ) of patients received single-muscle myectomy. Four patients $(8.5 \%)$ received myectomy of two muscles and one $(2.1 \%)$, three muscles (two rectus muscles in one eye and one rectus muscle in the other eye). Two patients received recession procedure on one additional muscle (both were on medial rectus). Fourteen patients (29.8\%) had extremely large angle (>40 PD; Table 1). 
Table 1 Patient summary

\begin{tabular}{|c|c|c|c|c|c|}
\hline Patient & $\begin{array}{l}\text { Deviation } \\
(\triangle, \text { prism diopter })\end{array}$ & Surgical correction & Outcome & Further surgery & Final outcome \\
\hline 1 & RHo30 & RIR myectomy & Orthotropia & & No diplopia \\
\hline 2 & RHo30 & RIR myectomy & Orthotropia & & No diplopia \\
\hline 3 & LHo30 & LIR myectomy & Orthotropia & & No diplopia \\
\hline 4 & LHo50 & LIR myectomy & Orthotropia & & No diplopia \\
\hline 5 & RET65 & BMR myectomy & Orthotropia & & No diplopia \\
\hline 6 & RXT30/RHo30 & RIR myectomy/RLR myectomy & Orthotropia & & No diplopia \\
\hline 7 & RHo30 & RIR myectomy & Orthotropia & & No diplopia \\
\hline 8 & RET45 & RMR myectomy & Orthotropia & & No diplopia \\
\hline 9 & LHo50 & LIR myectomy & LHo18 & No further surgery & LHo18 \\
\hline 10 & LH35 & LSR myectomy & Orthotropia & & No diplopia \\
\hline 11 & RH35 & RSR myectomy & Orthotropia & & No diplopia \\
\hline 12 & LHo38 & LIR myectomy & Orthotropia & & No diplopia \\
\hline 13 & RH40 & RSR myectomy & Orthotropia & & No diplopia \\
\hline 14 & LHo40 & LIR myectomy & LH40 & No further surgery & LH40 \\
\hline 15 & RHo26 & RIR myectomy & Orthotropia & & No diplopia \\
\hline 16 & RET62 & $\begin{array}{l}\text { RMR myectomy/LMRRC } \\
\text { 9-10 mm }\end{array}$ & Orthotropia & & No diplopia \\
\hline 17 & LHo60 & LIR myectomy & Orthotropia & & No diplopia \\
\hline 18 & LHo43 & LIR myectomy & Orthotropia & & No diplopia \\
\hline 19 & LHo55 & LIR myectomy & LH14 & LSR recession $5 \mathrm{~mm}$ & No diplopia \\
\hline 20 & RHo28 & RIR myectomy & Orthotropia & & No diplopia \\
\hline 21 & LHo30 & LIR myectomy & Orthotropia & & No diplopia \\
\hline 22 & LET30 & LMR myectomy & Orthotropia & & No diplopia \\
\hline 23 & RHo55 & RIR myectomy & RH23 & No further surgery & $\mathrm{RH} 23$ \\
\hline 24 & LHo26 & LIR myectomy & Orthotropia & & No diplopia \\
\hline 25 & LHo27 & LIR myectomy & Orthotropia & & No diplopia \\
\hline 26 & RHo30 & RIR myectomy & RHo20 & RIR re-myectomy & No diplopia \\
\hline 27 & LH52/LXT25 & LSR myectomy/LLR myectomy & Orthotropia & & No diplopia \\
\hline 28 & LH30 & LSR myectomy & Orthotropia & & No diplopia \\
\hline 29 & RET30 & RMR myectomy & RET20 & RMR re-myectomy & No diplopia \\
\hline 30 & LET66 & LMR myectomy/RMR RC8mm & Orthotropia & & No diplopia \\
\hline 31 & RHo35 & RIR myectomy & Orthotropia & & No diplopia \\
\hline 32 & LET50/RHo30 & BMR myectomy/RIR myectomy & Orthotropia & & No diplopia \\
\hline 33 & LHo30 & LIR myectomy & Orthotropia & & No diplopia \\
\hline 34 & RET35 & RMR myectomy & Orthotropia & & No diplopia \\
\hline 35 & LHo35 & LIR myectomy & Orthotropia & & No diplopia \\
\hline 36 & RHo38 & RIR myectomy & RHo16 & LSR recession $5.5 \mathrm{~mm}$ & No diplopia \\
\hline 37 & $\mathrm{RET}>50$ & RMR myectomy & Orthotropia & & No diplopia \\
\hline 38 & LET27 & LMR myectomy & Orthotropia & & No diplopia \\
\hline 39 & LHo40 & LIR myectomy & Orthotropia & & No diplopia \\
\hline 40 & LHo35 & LIR myectomy & Orthotropia & & No diplopia \\
\hline 41 & LHo30 & LIR myectomy & Orthotropia & & No diplopia \\
\hline 42 & RHo45 & RIR myectomy & RHo 37 & $\begin{array}{l}\text { RIR re-myectomy/LSR } \\
\text { recession } 6.5 \mathrm{~mm}\end{array}$ & No diplopia \\
\hline 43 & LET30 & LMR myectomy & RET25 & RMR myectomy & No diplopia \\
\hline 44 & RHo35 & RIR myectomy & RHo28 & RIR re-myectomy & RHo25 \\
\hline 45 & RHo40 & RIR myectomy & Orthotropia & & No diplopia \\
\hline 46 & RHo40 & RIR myectomy & Orthotropia & & No diplopia \\
\hline 47 & RHo35 & RIR myectomy & Orthotropia & & No diplopia \\
\hline
\end{tabular}

Abbreviations: LLR, left lateral rectus; LMR, left medial rectus; LSR, left superior rectus; LXT, left exotropia; MR, medial rectus; RC, recession; RET, right esotropia; RH, right hypertropia; RHo, right hypotropia; RIR, right inferior rectus; RMR, right medial rectus; RSR, right superior rectus; RXT, right exotropia; XT, exotropia.

Postoperatively, $78.7 \%(37 / 47)$ of patients reported no diplopia within central $30^{\circ}$ field and in reading position. Of the 10 patients who had persistent diplopia, seven $(70 \%)$ had under-correction of the deviation, while three (30\%) had overcorrection.
Seven patients opted to receive a second operation. Out of the six under-corrected patients, three underwent redetachment of the same muscles (case 26, 29, and 44), two received recession or myectomy of the antagonist of the yoke muscle (cases 36 and 43 respectfully) and one 


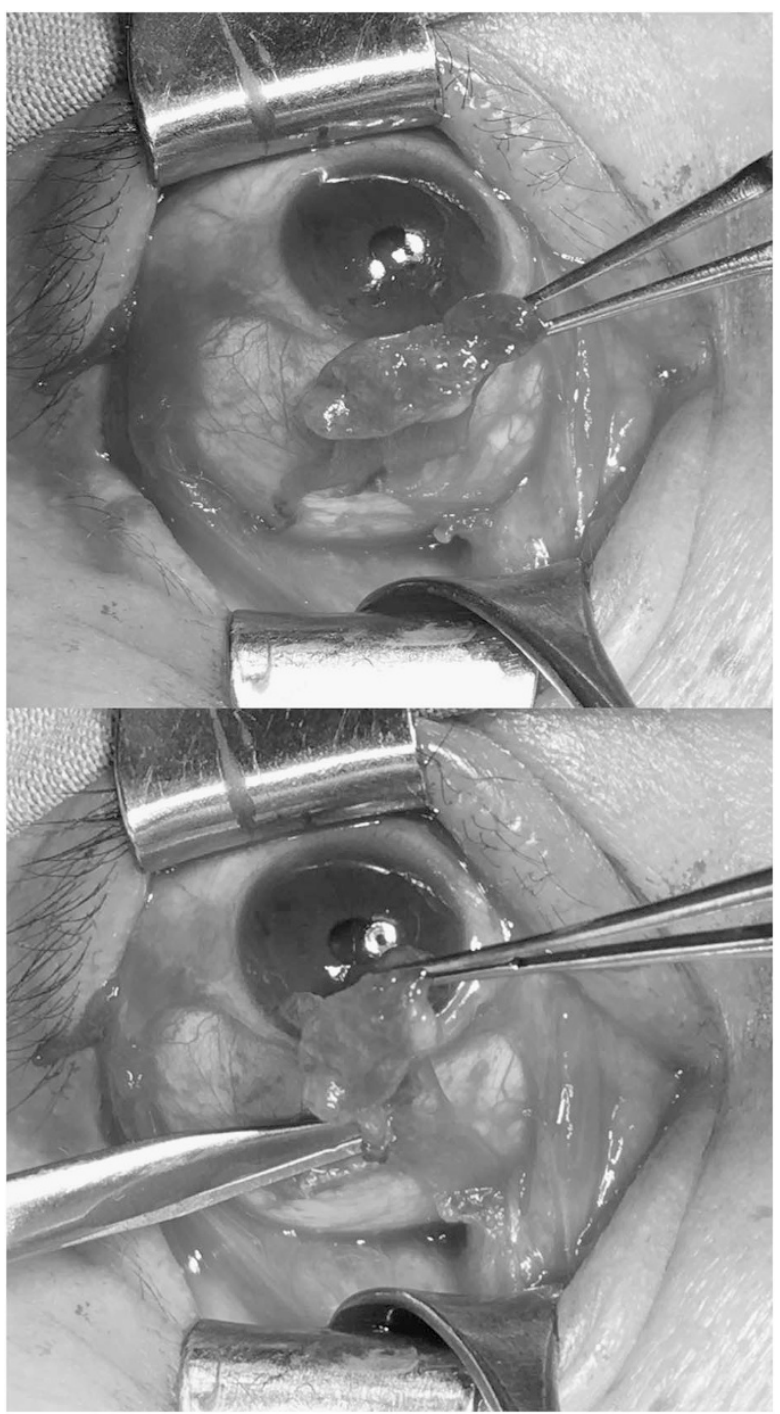

Figure 1 Myectomy of the rectus muscle. (upper) Myectomy procedure was done to detach the entire muscle at the muscle belly. (lower) The entire muscle stump was retrieved and removed from the insertion.

underwent re-detachment of the same muscle and concomitant recession of the antagonist of the yoke muscle (case 42). Recession of the antagonist in the same eye was performed in one re-operated case with overcorrection (case 19; Table 1). After the second operation, one patient with re-detachment of the same muscle reported persisitent diplopia. In this particular patient, severe fibrosis and adhesion of inferior rectus to surrounding tissues and eye globe was noted during the re-detachment procedure. The overall success rate for the second surgery was $85.7 \%(6 / 7)$ in reoperation group. The surgical success after re-detachment was 75\% (3/4). The overall success rate in this study group was $91.5 \%$

(Table 2).
Table 2 Surgical outcomes after rectus muscle myectomy

\begin{tabular}{lrrc}
\hline & Number & Success number & Success rate \\
\hline First surgery & & & \\
$\quad$ Single-muscle surgery & 40 & 30 & $75.0 \%$ \\
$\quad$ Multiple-muscle surgery & 7 & 7 & $100 \%$ \\
Overall & 47 & 37 & $78.7 \%$ \\
Second surgery & & & \\
$\quad$ Single-muscle surgery & 6 & 5 & $83.3 \%$ \\
$\quad$ Multiple-muscle surgery & 1 & 1 & $100 \%$ \\
Overall & 7 & 6 & $85.7 \%$ \\
& & & \\
Overall surgeries & & & \\
$\quad$ Single-muscle surgery & 40 & 36 & $90.0 \%$ \\
$\quad$ Multiple-muscle surgery & 7 & 7 & $100 \%$ \\
Overall & 47 & 43 & $91.5 \%$ \\
\hline
\end{tabular}

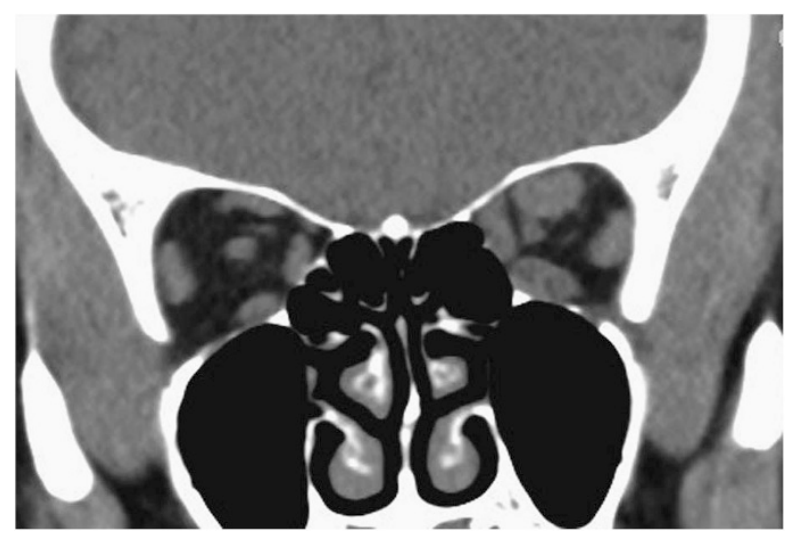

Figure 2 CT image of the patient with manifested overcorrection after myectomy. Significant enlargement of both left inferior rectus and left superior rectus muscles in the CT image of the patient with manifested overcorrection after myectomy (case 19).

Review of CT images of all patients showed markedly enlarged targeted rectus muscles. In addition, we also identified significant enlargement of the antagonist rectus muscles in the CT images of three patients who manifested overcorrection after myectomy (cases 14, 19, and 23; Figure 2). Severely restricted muscle movement and incomitance toward the opposite side were demonstrated in all patients with large-angle strabismus before the surgery. Significant improvement of muscle movement and incomitance toward opposite side was noted for all patients postoperatively. Some patients experienced slight limitation and incomitance toward the action of operated rectus muscle. However, those with functional binocular vision within central $30^{\circ}$ field and reading position were all satisfied with the surgical outcomes.

Forty patients were included in the calculation of the effectiveness of myectomy of a single restricted muscle 
Table 3 Effectiveness of isolated muscle detachment

\begin{tabular}{lccc}
\hline Muscle & Number & Prism diopters & Range \\
\hline Inferior rectus & 30 & $34.6 \pm 8.3$ & $26-60$ \\
Medial rectus & 6 & $34.3 \pm 2.1$ & $27-45$ \\
Superior rectus & 4 & $32.5 \pm 5.0$ & $30-40$ \\
Total & 40 & $34.3 \pm 7.7$ & $26-60$ \\
\hline
\end{tabular}

(Table 3). Only those who achieved no diplopia after a single-muscle myectomy were included for statistics. Patients with additional recession of one or more muscles were excluded from the calculation. The mean correction of deviation with a single-muscle myectomy was $34.3 \pm 7.7$ PD, ranging from 26 to 60 PD. All three groups of muscles had similar postoperative correction after myectomy (Table 3). The most commonly operated single muscle was inferior rectus (30 cases), followed by medial rectus (six cases) and superior rectus (four cases).

The mean preoperative exophthalmos was

$17.7 \pm 2.1 \mathrm{~mm}$. Postoperative exophthalmos was averaged $17.4 \pm 1.9 \mathrm{~mm}$. The differences had no statistical significance.

\section{Discussion}

Graves' orbitopathy is considered as one of the most complicated ophthalmological diseases. Infiltration of the inflammatory cells into the extraocular muscles change not only the elasticity but also the strength of the muscles, causing unpredictable surgical results regardless of careful planning. Up to date, no nomogram based on deviation can be established for Graves'-related strabismus. Prendiville suggested that in cases of vertical deviation, restriction of duction is more of a predicting factor for surgical success than the actual deviation. ${ }^{10}$ Nguyen et $a l^{6}$ showed that restoration of limited duction can achieve a higher success rate $(74 \%)$ than correction of deviation (44\%). Adjustable suture that addresses duction has become the most commonly practiced way in tackling with Graves'-related strabismus. ${ }^{15-17,19-22}$ However, modifications are still underway for large-angle strabismus because it often involves more advanced stage of the disease and the results were still unsatisfactory. Dal Canto et al, using an intraoperative relaxed muscle positioning technique, were able achieve an $87.5 \%$ excellent final outcome in a retrospective consecutive case series. ${ }^{17}$ However, their report was not limited to largeangle Graves'-related strabismus. Zoumalan et al ${ }^{16}$ claimed that tenon recession is an effective adjunct to improve the surgical outcome of large-angle strabismus, resulting in an overall success rate of $84.6 \%$. However, the correction for vertical strabismus achieved only $43 \%$ success.
In this study, we have shown that a novel approach to large-angle Graves'-related strabismus with myectomy of the restricted extraocular muscles can provide as a simple and effective option to this troublesome situation. The operative technique described herein is straight forward and highly replicable, as proven by the high success rate $(78.7 \%)$ in the cases with primary myectomy procedure.

We have developed this procedure specifically for large-angle Graves'-related strabismus because of the limited space often encountered during the surgery. ${ }^{23}$ In advanced Graves' orbitopathy, even though we choose to perform the surgery after 3 month of stability, when the orbital inflammation has quieted down, the muscles are still markedly enlarged and tightly packed between the sclera and the orbital wall. The markedly enlarged muscles were tightly pushed against the orbital wall in that adjustable sutures may not provide enough release. Even though the adjustable sutures for the correction of small-angle Graves'-related strabismus in our experience have mostly been successful, the results in large-angle strabismus were less satisfactory. Postoperative adjustments in these cases often involve total release of the sutures in order to achieve an end stage of no diplopia. At times, even such extreme maneuver was still inadequate. In addition, if detachment was done at the insertion, the muscle may simply not have enough leeway to recess to its most relaxed position. This condition may explain why seven of our cases had encountered under-correction despite an initial myectomy.

When under-correction was encountered, we suspect that the residual rectus muscle had reattached to the underlying sclera due to the tight orbit, causing a consecutive muscle restriction in some cases.

Re-detachment with further myectomy procedure allows the surgeon to reach further behind the second insertion to re-detach the muscle. In those under-corrected cases with restriction of the antagonist of the yoke muscle, we opted to perform recession or myectomy of the antagonist of the yoke muscle instead of re-detachment. Both of these patients (one with superior rectus recession and one with medial rectus myectomy) achieved a final result of no diplopia within central $30^{\circ}$ field and in reading position. It is not clear whether adjustable sutures have enough holding force to counteract the increased elastic force of the muscles, as inferred by the common complication of late overcorrection often observed in inferior rectus recession. ${ }^{10,20}$ In our study, without any suture in situ, myectomy of the restricted muscle achieved good surgical results without overcorrection in most of the cases. A possible explanation is that the enlarged residual muscle belly reattaches to the underlying sclera more effectively due to the tight orbital space encountered in 
these more advanced disease, which in a way counteracts the antagonist muscle in the same eye.

Some eye muscle specialists may be concerned about the incomitance after myectomy. In this study, severely restricted muscle movement and incomitance toward opposite side was recognized for all patients with largeangle strabismus before operations. Most patients also showed overaction of operated rectus muscle and complained diplopia when looking toward the direction of operated rectus muscle. Our results showed the improvement of muscle movement and incomitance toward opposite side for all patients after myectomy. The patients may have experienced slight limitation and incomitance toward the action of operated rectus muscle. Fortunately, the patients with success results remained no diplopia in central $30^{\circ}$ field and reading position and were satisfied with the surgical results. Only very few patients experienced extreme downgaze diplopia when going downstairs and needed slight head-position adjustment.

The effectiveness of a single muscle myectomy in the absence of restriction of the antagonists is approximately 30 PD. The degree of correction is consistent among inferior rectus, medial rectus, and superior rectus. We believe this procedure is consistently effective because after the myectomy, the muscle may fall to its most relaxed position and to attach to the underlying sclera at the new position. In the presence of possible restricted antagonists, the correction can be as much as $80 \mathrm{PD}$, as exemplified in our three overcorrection cases (cases 14, 19, and 23). In our radiology review, we were able to find significant enlargement of the antagonist rectus muscles in the CT images of these three patients. These unforeseen large overcorrections may possibly be prevented had the orbital CT been evaluated more carefully before the surgery. Immediately following the surgery, it may be possible to minimize the complication by applying tractional suture to the limbus opposite the direction of the myectomy for a few days until the muscle is rightly attached to underlying sclera.

As we know, large inferior rectus recessions or myectomy may result in lower lid retraction. ${ }^{18}$ For patients undergoing inferior rectus myectomy, we performed simultaneous detachment of the fascia of the capsulopalpebral head during inferior rectus myectomy to minimize the possibility of lower lid retraction. ${ }^{18}$ In this study, some patients showed minimal lower lid retraction without further treatment after inferior rectus myectomy. Only three patients with very large hypotropia suffered from disfiguring lower-lid retraction after inferior rectus myectomy and underwent lower-lid retraction correction with hard-palate graft.

Since proptosis is one of the disfiguring complications of thyroid-related orbitopathy, there are concerns that myectomy of the restricted extraocular muscles may exacerbate exophthalmos. However, no statistically significant proptotic changes were found in our patients. It is therefore safe to perform myectomy should changes in proptosis be a concern.

It is interesting to note that in the large-angle Graves'-related strabismus recruited in our study, the involvement of inferior rectus (34) far outnumbered medial rectus (9) and superior rectus (6) (Table 1). Even though it is well known that the inferior rectus is most commonly involved in Graves'-related strabismus, the ratio appears out of proportion. In Yan's report that addressed large-angle strabismus with Graves' ophthalmopathy, the ratio between involved inferior rectus, medial rectus, and superior rectus were $16: 21$ : $15 .^{24}$ Zoumalan et $a l^{16}$ reported that horizontal deviation was more common than vertical deviation but the muscles were not specified. Also, it is worthwhile to report that the deviations encountered in our esotropic cases tend to be much larger. It may be due to cases with extreme bilateral enlarged medial rectus, which need more aggressive treatment.

One of the limitations in this case series is the lack of a control group. However, similar studies utilizing different surgical methods can be used for comparison. ${ }^{6,17} \mathrm{We}$ believe the difficult situation of a large-angle Graves'related strabismus deserves to summon as many surgical solutions as possible. A multi-center prospective comparative study may be of value in establishing surgical protocols for this sophisticated ophthalmological condition in Graves' orbitopathy.

Our novel technique of rectus myectomy for large-angle Graves'-related strabismus allows consistent and excellent results and low reoperation rates. The simplicity of the surgical technique provides minimal intraoperative and postoperative manipulation and allows high reproducibility even in subsequent surgeries. It can be used in single-muscle operation or multiple-muscle operation.

\section{Summary}

What was known before

- For large-angle strabismus, especially with single-rectus muscle restriction without much of the contralateral antagonist muscle involvement, surgical approaches have mainly been multiple-muscle recession with adjustable sutures.

What this study adds

- In this study, we found that complete rectus muscle myectomy technique is effective and predictable in the treatment of large-angle strabismus in patients with Graves' ophthalmopathy.

\section{Conflict of interest}

The authors declare no conflict of interest. 


\section{References}

1 Bartley GB, Gorman CA. Diagnostic criteria for Graves' ophthalmopathy. Am J Ophthalmol 1995; 119(6): 792-795.

2 Bahn RS, Heufelder AE. Mechanisms of disease: pathogenesis of Graves' ophthalmopathy. N Engl J Med 1993; 329: 1468-1475.

3 Flanders M, Hastings M. Diagnosis and surgical management of strabismus associated with thyroid-related orbitopathy. J Pediatr Ophthalmol Strabismus 1997; 34(6): 333-340.

4 Lueder GT, Scott WE, Kutschke PJ, Keech RV. Long-term results of adjustable suture surgery for strabismus secondary to thyroid ophthalmopathy. Ophthalmology 1992; 99(6): 993-997.

5 Inoue Y, Tsuboi T, Kouzaki A, Maeda T, Inoue T. Ophthalmic surgery in dysthyroid ophthalmopathy. Thyroid 2002; 12(3): 257-263.

6 Nguyen VT, Park DJ, Levin L, Feldon SE. Correction of restricted extraocular muscle motility in surgical management of strabismus in graves' ophthalmopathy. Ophthalmology 2002; 109(2): 384-388.

7 Evans D, Kennerdell JS. Extraocular muscle surgery for dysthyroid myopathy. Am J Ophthalmol 1983; 95(6): 767-771.

8 Mocan MC, Ament C, Azar NF. The characteristics and surgical outcomes of medial rectus recessions in Graves' ophthalmopathy. J Pediatr Ophthalmol Strabismus 2007; 44(2): 93-100, quiz 18-9.

9 Ellis FD. Strabismus surgery for endocrine ophthalmopathy. Ophthalmology 1979; 86(12): 2059-2063.

10 Prendiville P, Chopra M, Gauderman WJ, Feldon SE. The role of restricted motility in determining outcomes for vertical strabismus surgery in Graves' ophthalmology. Ophthalmology 2000; 107(3): 545-549.

11 Scott WE, Thalacker JA. Diagnosis and treatment of thyroid myopathy. Ophthalmology 1981; 88(6): 493-498.

12 Mourits MP, Koorneef L, van Mourik-Noordenbos AM, van der Meulen-Schot HM, Prummel MF, Wiersinga WM et al. Extraocular muscle surgery for Graves' ophthalmopathy: does prior treatment influence surgical outcome? $\mathrm{Br} J$ Ophthalmol 1990; 74(8): 481-483.
13 Rosenbaum AL, Metz HS, Carlson M, Jampolsky AJ. Adjustable rectus muscle recession surgery. A follow-up study. Arch Ophthalmol 1977; 95(5): 817-820.

14 Kraus DJ, Bullock JD. Treatment of thyroid ocular myopathy with adjustable and nonadjustable suture strabismus surgery. Trans Am Ophthalmol Soc 1993; 91: 67-79 discussion -84 .

15 Fells P, Kousoulides L, Pappa A, Munro P, Lawson J. Extraocular muscle problems in thyroid eye disease. Eye (Lond) 1994; 8(Pt 5): 497-505.

16 Zoumalan CI, Lelli Jr GJ, Kazim M. Tenon recession: a novel adjunct to improve outcome in the treatment of large-angle strabismus in thyroid eye disease. Ophthal Plast Reconstr Surg 2011; 27(4): 287-292.

17 Dal Canto AJ, Crowe S, Perry JD, Traboulsi EI. Intraoperative relaxed muscle positioning technique for strabismus repair in thyroid eye disease. Ophthalmology 2006; 113(12): 2324-2330.

18 Liao SL, Shih MJ, Lin LL. A procedure to minimize lower lid retraction during large inferior rectus recession in graves ophthalmopathy. Am J Ophthalmol 2006; 141(2): 340-345.

19 Kose S, Uretmen O, Emre S, Pamukcu K. Recession of the inferior rectus muscle under topical anesthesia in thyroid ophthalmopathy. J Pediatr Ophthalmol Strabismus 2002; 39(6): 331-335.

20 Boulos PR, Hardy I. Thyroid-associated orbitopathy: a clinicopathologic and therapeutic review. Curr Opin Ophthalmol 2004; 15(5): 389-400.

21 Weston B, Enzenauer RW, Kraft SP, Gayowsky GR. Stability of the postoperative alignment in adjustable-suture strabismus surgery. J Pediatr Ophthalmol Strabismus 1991; 28(4): 206-211.

22 Sharma P, Reinecke RD. Single-stage adjustable strabismus surgery for restrictive strabismus. J AAPOS 2003; 7(5): 358-362.

23 Schotthoefer EO, Wallace DK. Strabismus associated with thyroid eye disease. Curr Opin Ophthalmol 2007; 18(5): 361-365.

24 Yan J, Zhang H. The surgical management of strabismus with large angle in patients with Graves' ophthalmopathy. Int Ophthalmol 2008; 28(2): 75-82. 